\title{
Finding new signature effects on galactic dynamics to constrain Bose-Einstein-condensed cold dark matter
}

\author{
Tanja Rindler-Daller* and Paul R. Shapiro \\ Department of Astronomy and Texas Cosmology Center, \\ The University of Texas at Austin, 2515 Speedway C1400, Austin, TX 78712, USA
}

(Dated: October 31, 2018)

\begin{abstract}
If cosmological cold dark matter (CDM) consists of light enough bosonic particles that their phasespace density exceeds unity, they will comprise a Bose-Einstein condensate (BEC). The nature of this BEC-CDM as a quantum fluid may then distinguish it dynamically from the standard form of CDM involving a collisionless gas of non-relativistic particles that interact purely gravitationally. We summarize some of the dynamical properties of BEC-CDM that may lead to observable signatures in galactic halos and present some of the bounds on particle mass and self-interaction coupling strength that result from a comparison with observed galaxies.
\end{abstract}

PACS numbers: 95.35.+d; 95.30.Cq; 98.62.Gq; 03.75.Nt

\section{INTRODUCTION}

Astronomical observations suggest the presence of nonbaryonic, non-relativistic ('cold') dark matter (DM), comprising around 23 per cent of the energy density in the Universe. The particle nature of dark matter remains elusive, however, despite ongoing efforts to detect it directly in search experiments or indirectly via imprints on astrophysical observations. In large N-body simulations of structure formation, cold dark matter (CDM) has been modeled as a collisionless gas, which only interacts gravitationally. We refer to this as standard CDM. Despite the successes in reproducing the large-scale structure, as well as reproducing flat rotation curves at the outskirts of galaxies, standard CDM seems to be in conflict with observations of galactic small-scale properties. These are notably the overabundance of subhalos around big hosts of Milky-Way size and beyond, and the failure to reproduce flat cores in the centers of dark-matter dominated dwarf and LSB galaxies. Both features apparently contradict astronomical observations, and have been the subject of active research in the past decade. One approach has been to study the combined effects of the collisionless CDM component and the dissipative baryonic component, to see if the dissipative hydrodynamics of the latter can affect a cure. Another possible solution has been to go beyond the simplifying assumption that the DM particles are cold and/or collisionless. As a result, there has been a recent revival of investigations of structure formation with non-standard dark matter candidates, like warm dark matter e.g. Schneider et al. 29], self-interacting fermionic dark matter (SIDM) e.g. Ahn \& Shapiro [1], Koda \& Shapiro [19] and Bose-Einsteincondensed dark matter (or scalar-field dark matter), e.g. Woo \& Chiueh [39] and Suárez \& Matos [35], all proposals of which are able to suppress the formation of self-bound structures below a certain scale, as well as to

\footnotetext{
* Invited plenary speaker at the IV International Meeting on Gravitation and Cosmology, Guadalajara, MX, May 21-25, 2012
}

flatten central profiles, depending on the respective particle parameters.

In this paper, we will address Bose-Einstein-condensed DM (BEC-CDM). We are particularly interested in a class of models describing self-interacting bosonic dark matter, the particles of which are so light that they collectively occupy their ground state below a certain temperature, forming a Bose-Einstein condensate (BEC) in the early Universe. Then, this state can be well described by a scalar field, the so-called wavefunction of the condensate. We will assume that the wavefunction respects a $U(1)$-symmetry, such that the number of particles is conserved. There is thus no self-annihilation of DM in this scenario, in contrast to models considered for instance by Tkachev [36]. Recent advances in particle theory predict the generic existence of bosons as light as or much lighter than the QCD axion - the 'classic' bosonic DM candidate, values ranging from about $10^{-33}$ to $\gtrsim 10^{-5} \mathrm{eV} / \mathrm{c}^{2}$, which can serve as the CDM in the Universe (see e.g. Günther \& Zhuk [15], Carroll [8], Arvanitaki et al. [4]). The theoretical description of these very light bosons in terms of scalar fields leads to halo dynamics which can be understood as the solution of nonlinear wave equations (e.g. Alcubierre et al. 2], Chavanis [10], Matos \& Ureña-López 24], Sin [33], Ureña-López \& Guzmán 37]), in contrast to the $N$-body dynamics of standard CDM. As a matter of fact, much less investigation has been pursued in the literature so far, in particular with respect to the nonlinear stages of structure formation for this type of DM. Therefore, we are in need of a better understanding of how much of the parameter space of this form of dark matter is able to reproduce the successes, while resolving the failures, of standard CDM.

In what follows, we will summarize some of the recent work in this field, highlighting some of our own, with apologies for the fact that length limitations prevent us from attempting a more comprehensive review. In Section III. we will describe the basic equations which govern BEC-CDM dynamics, showing how its quantum nature leads to fluid behavior. We will then distinguish two regimes according to the strength of the particle self- 
interaction, and state how the characteristic size and mass of structures that form gravitationally from this form of DM are related to the particle mass $m$ and selfinteraction coupling strength $g$. We also present the virial theorem for isolated BEC-CDM halos. In Section III, we summarize how the equilibrium structure of BEC-CDM halos can distinguish them from halos in standard CDM, for halos with and without rotation. We show that halos in the BEC-CDM model typically rotate with enough angular momentum that, if they are of the type supported against gravitational collapse by self-interaction pressure and rotation, quantum vortices are likely to form, which can affect halo density profiles. We also summarize a few examples in which BEC-CDM can be distinguished by its effect on baryonic structures. In Section IV] we summarize the bounds on particle mass and coupling strength that follow from the requirement that halos or halo cores not exceed some characteristic size, if halos or their cores are treated as isolated, equilibrium structures. Finally, in Section $\square$ we argue that, for BEC-CDM to reproduce the full range of structure scales found in our Universe (as in standard CDM), we must go beyond the modeling of individual objects in static equilibria to account for continuous mass infall and its dynamical consequences.

\section{QUANTUM-COHERENT DARK MATTER UNDER NEWTONIAN GRAVITY}

\section{A. Fundamental properties}

The dynamical description of dark matter within galactic halos usually involves small gravitational and velocity fields. With regard to BEC-CDM, it has proved advantageous to consider the non-relativistic SchrödingerPoisson, or Gross-Pitaevskii-Poisson (GPP) system of equations of motion for the dark matter BEC wavefunction $\psi$, as follows

$$
\begin{gathered}
i \hbar \frac{\partial \psi}{\partial t}=-\frac{\hbar^{2}}{2 m} \Delta \psi+m \Phi \psi+g|\psi|^{2} \psi, \\
\Delta \Phi=4 \pi G m|\psi|^{2} .
\end{gathered}
$$

The terms on the rhs in (1), which govern the evolution, are due to the quantum-kinetic energy, the gravitational potential $\Phi$, and the self-interaction of identical bosons. The latter has been included in the usual way in terms of an effective interaction potential $g|\psi|^{4} / 2$ with coupling constant (or self-interaction strength) $g$. The possibly complicated particle interactions are simplified this way in the low-energy limit of a dilute gas: disregarding higher than 2-body interactions, the cross section for elastic scattering of indistinguishable bosons becomes constant in the low-energy limit,

$$
\sigma=8 \pi a_{s}^{2}
$$

with the s-wave scattering length $a_{s}$. The coupling constant of the effective interaction is then given by

$$
g=4 \pi \hbar^{2} a_{s} / m
$$

We shall note that the above GP equation is strictly valid only for dilute systems, which means that $a_{s}$ must be much smaller than the mean interparticle distance, i.e. $a_{s} \ll n^{-1 / 3}$. Also, we are restricted to $g \geq 0$ $\left(a_{s} \geq 0\right)$, because models described by (1) with negative self-interaction coupling $g$ are not able to provide stable structures, since the resulting negative pressure works in favor of gravity. If we assume that all of the DM is in the condensed state, the number and mass density of DM in the halo is given by $n(\mathbf{r})=|\psi|^{2}(\mathbf{r})$ and $\rho(\mathbf{r})=m n(\mathbf{r})$, respectively.

The equations can be written in fluid-like form by inserting into (11) the polar decomposition of the wavefunction (applied early by Madelung [23] to the free-particle Schrödinger equation),

$$
\psi(\mathbf{r}, t)=|\psi|(\mathbf{r}, t) e^{i S(\mathbf{r}, t)}=\sqrt{\frac{\rho(\mathbf{r}, t)}{m}} e^{i S(\mathbf{r}, t)},
$$

resulting in the momentum and continuity equations,

$$
\rho \frac{\partial \mathbf{v}}{\partial t}+\rho(\mathbf{v} \cdot \nabla) \mathbf{v}=-\rho \nabla Q-\rho \nabla \Phi-\nabla P_{S I},
$$

$$
\frac{\partial \rho}{\partial t}+\nabla \cdot(\rho \mathbf{v})=0
$$

with the bulk velocity $\mathbf{v}=\hbar \nabla S / m$. The gradient of

$$
Q=-\hbar^{2} \Delta \sqrt{\rho} /\left(2 m^{2} \sqrt{\rho}\right)
$$

gives rise to what is often called 'quantum pressure', an additional force on the rhs of equ.(6), which basically stems from the quantum-mechanical uncertainty principle. The particle self-interaction, on the other hand, gives rise to a pressure of polytropic form

$$
P_{S I}=g \rho^{2} /\left(2 m^{2}\right) .
$$

The quantum-kinetic term provides an important characteristic length scale, as follows: with $\Delta$ having a dimension of $\mathrm{L}^{-2}$ and changing to the momentum representation, one can easily see that the characteristic length is essentially nothing but the de Broglie length of the bosons

$$
L \sim \lambda_{d e B}=h / p=h /(m v) .
$$

Since we will consider virialized, isolated objects, it makes sense to use the corresponding virial velocity in this expression for $\lambda_{d e B}$, assuming that the particles stay in their Bose-Einstein-condensed state after virialization, which is the case for the model parameters we are going to encounter in this paper (see also Section IV). BEC$\mathrm{CDM}$ without self-interaction, $P_{S I}=0$, was termed 
TABLE I. Lower bound on the boson mass, provided by the 'Fuzzy Dark Matter' regime, or TYPE I BEC-CDM, for different cosmological structures

\begin{tabular}{llll}
\hline \hline & $\begin{array}{l}\text { halo mass } \\
{\left[M_{\odot}\right]}\end{array}$ & $\begin{array}{c}\text { size } \\
{[\mathrm{kpc}]}\end{array}$ & $\begin{array}{l}\text { boson mass } \\
{[\mathrm{eV}]}\end{array}$ \\
\hline Milky Way (MW) & $10^{12}$ & 100 & $1.066 \cdot 10^{-25}$ \\
Dwarf Galaxy (DG) & $10^{10}$ & 10 & $3.371 \cdot 10^{-24}$ \\
Dwarf Spheroidal $(\mathrm{dSph})$ & $10^{8}$ & 1 & $1.066 \cdot 10^{-22}$ \\
Minihalo $(\mathrm{MH})$ & $10^{6}$ & 0.1 & $3.371 \cdot 10^{-21}$ \\
\hline \hline
\end{tabular}

'Fuzzy Dark Matter' by Hu, Barkana \& Gruzinov [16]. In this review, we will call it BEC-CDM of TYPE I. In this regime, it is the quantum-kinetic term via (10) which determines the equilibrium size of self-gravitating objects - in order for $\lambda_{d e B}$ not to exceed a certain galactic length scale, the particle mass $m$ must be sufficiently large. Table \contains different typical halo sizes and the corresponding lower limits on $m$. However, it has been observed in the previous literature, e.g. Colpi, Shapiro \& Wasserman [12] and Lee \& Lim [21], and we confirm it as well, see equ.(13) and Section III, that a larger mass $m$ than inferred from (10) can result in stable structures of the same given size, if self-interaction is included. In fact, considering the case in which the last term in (6) supports the system against gravitational collapse, while $Q=0$, we arrive at the opposite regime to TYPE I, which we call the Thomas-Fermi regime of BEC-CDM, or TYPE II for short. We note that this regime goes under many names; Goodman [13] calls it 'repulsive dark matter' (RDM), Peebles [26] speaks of 'fluid dark matter'. We also note that both regimes have already been considered for related models by Khlopov, Malomed \& Zeldovich [18], in studying gravitational instabilities of a primordially produced scalar field.

In the following, we will make use of convenient units, defined as in Rindler-Daller \& Shapiro (RDS) [28]:

$$
\begin{gathered}
m_{H} \equiv \frac{\hbar}{R^{2}(\pi G \bar{\rho})^{1 / 2}}=\frac{2 \hbar}{\sqrt{3 G}}(R M)^{-1 / 2}= \\
=1.066 \cdot 10^{-22}\left(\frac{R}{1 \mathrm{kpc}}\right)^{-1 / 2}\left(\frac{M}{10^{8} M_{\odot}}\right)^{-1 / 2} \mathrm{eV},
\end{gathered}
$$

and

$$
\begin{gathered}
g_{H} \equiv \hbar^{2} /\left(2 \bar{\rho} R^{2}\right)=2 \pi \hbar^{2} R /(3 M)= \\
=2.252 \cdot 10^{-62}\left(\frac{R}{1 \mathrm{kpc}}\right)\left(\frac{M}{10^{8} M_{\odot}}\right)^{-1} \mathrm{eV} \mathrm{cm}{ }^{3},
\end{gathered}
$$

with $c=1$. We described the meaning and significance of those parameters at length in RDS [28]. It shall be sufficient to re-iterate here that $m_{H}$ is the characteristic mass of a non-interacting particle whose de Broglie wavelength is comparable to the size of a given halo, see
Table II It is thus the smallest particle mass possible in order for quantum pressure to be solely responsible for holding that halo up against gravitational collapse. On the other hand, if there are density variations in the BEC fluid of scale length $R$, then $g_{H}$ is the coupling strength for which the quantum and self-interaction pressure force terms are equal.

It turns out that the TYPE II regime is a good approximation as long as $g / g_{H} \gg 2$ is fulfilled, as we demonstrated in 28]: to determine whether a BEC-CDM halo of a given size $R$ is of TYPE I or TYPE II, we have to compare the quantum pressure and self-interaction pressure terms in (6) to each other,

$$
|-\rho \nabla Q| /\left|-\nabla P_{S I}\right| \sim \hbar^{2} /\left(g \rho R^{2}\right) \sim 2 g_{H} / g \ll 1,
$$

from which the claim follows. We have shown in RDS [28], equ.(46), that the radius of a spherical halo is then related to the de-Broglie length of the boson according to

$$
R_{0}=\frac{\sqrt{3} \pi^{1 / 4}}{12}\left(\frac{g}{g_{H}}\right)^{1 / 2} \lambda_{d e B}
$$

Hence, since $g / g_{H} \gg 2, R_{0} \gg \lambda_{d e B}$ for TYPE II BECCDM halos.

\section{B. Stationary systems and virial equilibrium}

In the context of BEC-CDM in the GPP framework, equ.(11)-(2), stationary self-gravitating halos can be described by wavefunctions of the form

$$
\psi(\mathbf{r}, t)=\psi_{s}(\mathbf{r}) e^{-i \mu t / \hbar},
$$

where the conservation of particle number fixes $\mu$, the GP chemical potential. While $\psi$ evolves harmonically in time, the mass density $\rho=m\left|\psi_{s}\right|^{2}$ and, hence, the gravitational potential $\Phi$ are time-independent. Inserting this $\psi$ into (11) results in the time-independent GP equation with eigenvalues $\mu$,

$$
\left(-\frac{\hbar^{2}}{2 m} \Delta+g\left|\psi_{s}\right|^{2}+m \Phi\right) \psi_{s}=\mu \psi_{s}
$$

The time-independent part $\psi_{s}(\mathbf{r})$ itself can be decomposed as usual,

$$
\psi_{s}(\mathbf{r})=\left|\psi_{s}\right|(\mathbf{r}) e^{i S_{s}(\mathbf{r})}
$$

with both amplitude and phase depending here on position only. We will omit the subscript 's' in the forthcoming analysis. Systems obeying (15) can be studied via the corresponding GP energy functional given by

$$
\mathcal{E}[\psi]=\int_{V}\left[\frac{\hbar^{2}}{2 m}|\nabla \psi|^{2}+\frac{m}{2} \Phi|\psi|^{2}+\frac{g}{2}|\psi|^{4}\right] d^{3} \mathbf{r} .
$$

Inserting (16) into (17), the total energy can be written as

$$
E=K+W+U_{S I},
$$


with the total kinetic energy term

$$
\begin{gathered}
K \equiv \int_{V} \frac{\hbar^{2}}{2 m}|\nabla \psi|^{2} d^{3} \mathbf{r}= \\
=\int_{V} \frac{\hbar^{2}}{2 m^{2}}(\nabla \sqrt{\rho})^{2} d^{3} \mathbf{r}+\int_{V} \frac{\rho}{2} \mathbf{v}^{2} d^{3} \mathbf{r} \equiv K_{Q}+T .
\end{gathered}
$$

$K_{Q}$ accounts for the quantum-kinetic energy and $T$ for the bulk kinetic energy of the body, which comes in the form of rotation or internal motion. $K_{Q}$ has no classical counterpart, and is absent in the classical figures of equilibrium studied in the previous literature. Also, $K_{Q}$ is neglected in the TYPE II regime. The other terms in (18) are the gravitational potential energy

$$
W \equiv \int_{V} \frac{\rho}{2} \Phi d^{3} \mathbf{r}
$$

and the internal energy

$$
U_{S I} \equiv \int_{V} \frac{g}{2 m^{2}} \rho^{2} d^{3} \mathbf{r},
$$

which is determined by the particle interactions, and which we have defined essentially as $U_{S I}=\int P_{S I} d V$ with $P_{S I}$ in (9). The origin of $U_{S I}$ is due to the repulsive 2body elastic scattering of identical bosons, equ. (3). The above energy contributions enter the scalar virial theorem of an isolated (possibly rotating) BEC halo under self-gravity, which reads as

$$
2 K+W+3 U_{S I}=0
$$

As in classical gas dynamics, (22) can be derived by multiplying the equations of motion in fluid form, equ.(6), by $\mathbf{r}$ and integrating the resulting equation over volumes which enclose the system of interest. For an isolated body, a derivation involving a scaling argument was presented by Wang [38].

\section{SIGNATURE EFFECTS OF BEC-CDM ON HALOS AND HALO CORES}

\section{A. Sizes and density profiles}

The equilibrium density profiles of self-gravitating BEC-CDM halos are solutions of (1) or (6)-(9) respectively, along with (2). As an important result, they are universal in shape. Furthermore, BEC-CDM halos have a finite central density. In fact, this last feature has been one of the motivations in the previous literature to consider this form of DM as a solution to the cusp-core problem of dark matter dominated dwarf galaxies (DG) and dwarf-spheroidal (dSph) galaxies.

In the case of TYPE I, the density profile can only be determined numerically: it falls off as $r^{-4}$ for large $r$, but has no compact support. The radius which includes 99 per cent of the mass reads as

$$
R_{99}=9.9 \hbar^{2} /\left(G M m^{2}\right)
$$

(see Membrado, Pacheco \& Sañudo [25] for more details). For TYPE II, on the other hand, the equation of state reduces to an $(n=1)$-polytrope, with $P_{S I}$ in (9) and $Q=0$, having the well-known spherical density profile

$$
\rho^{S}(r)=\rho_{c}^{S} \operatorname{sinc}\left(\sqrt{4 \pi G m^{2} / g} r\right),
$$

with $\operatorname{sinc}(x) \equiv \sin (x) / x$ and the central density $\rho_{c}^{S}$. The corresponding halo radius is then given by

$$
R_{0}=\pi \sqrt{\frac{g}{4 \pi G m^{2}}},
$$

see e.g. Goodman [13] for more details.

In both regimes, TYPE I and TYPE II, the halo profile and size are determined by the DM particle parameters. Of course, this is also true for the intermediate regime: Chavanis \& Delfini [11] calculate numerical solutions for the mass-radius relationship, $R=R(M)$, which interpolate between TYPE I and II. However, for any given particle model, neither (23) and (25) nor the result of 11] are able to re-produce the fact that $R$ must increase with $M$ as we know from astronomical observations. The successful fitting of galaxy data using the associated rotation curves of TYPE I and TYPE II BEC-CDM halos by Arbey, Lesgourgues \& Salati [3] and Böhmer \& Harko 7] must thus be judged with this caveat in mind. It implies that it is necessary to go beyond the description of non-rotating halos in virial equilibrium, composed of a pure BEC-CDM fluid, if this DM model is to describe galactic structures successfully.

\section{B. Rotation and shape}

In RDS 28], we studied the properties of BEC-CDM halos in the TYPE II case, once rotation is taken into account. It is generally believed that tidal torques caused by large-scale structure give a halo most of its angular momentum in the early phases of halo collapse. This picture has been confirmed by cosmological N-body simulations of the standard CDM universe, which show that halos form with a net angular momentum such that the dimensionless ratio, the so-called spin parameter,

$$
\lambda=\frac{L|E|^{1 / 2}}{G M^{5 / 2}},
$$

where $L$ is the total angular momentum and $E$ the total energy of the halo, has typical values in the range of $[0.01,0.1]$ with a median value $\simeq 0.05$ (see e.g. Barnes \& Efstathiou [5]). The degree of rotational support is thus very small for the CDM halos which surround galaxies. We will be interested in the case where the BEC nature of DM affects small-scale structure and the internal 
dynamics of halos, while large-scale structure formation shall follow the $\Lambda$ CDM model to a great extent. Therefore, we adopt the above range of spin parameters for BEC-CDM halos, too.

We describe the effect of rotation on the structure of BEC-CDM halos by two approximations which are based upon the classic models of rotating figures of equilibrium (see e.g. Chandrasekhar [9]). The simplest description assumes that the halos are Maclaurin spheroids, which are axisymmetric, oblate, and homogeneous. Not only is this model fully analytical, it also provides a convenient background solution to perturb in determining if and when quantum vortex formation is energetically favored. In the absence of quantum vortices, however, BEC-CDM is irrotational, while the Maclaurin spheroid model assumes uniform rotation. Of course, this irrotationality can be broken locally in the fluid, by creating quantum vortices, if the amount of angular momentum exceeds a certain minimum, as shown below. In the limit of large enough angular momentum that a vortex lattice develops, in fact, uniform rotation is a good approximation, and so will the Maclaurin spheroids be. More generally, to account for irrotationality, we also consider a second model, that of irrotational Riemann-S ellipsoids. Since the classic solution for Riemann-S ellipsoids is homogeneous, however, we account for the $(n=1)$-polytropic nature of TYPE II BEC-CDM by adopting the solution derived by Lai, Rasio \& Shapiro (LRS) [20] for compressible Riemann-S ellipsoids, based on the 'ellipsoidal approximation' which assumes self-similar ellipsoidal density strata.

We denote the semi-axes of those bodies along $(x, y, z)$ as $\left(a_{1}, a_{2}, a_{3}\right)$. Maclaurin spheroids fulfill $a_{1}=a_{2}>a_{3}$ with eccentricity $e=\left(1-\left(a_{3} / a_{1}\right)^{2}\right)^{1 / 2}$. Using (22), we can determine how the (mean) radius $R=\left(a_{1} a_{2} a_{3}\right)^{1 / 3}$ and the spin parameter of such a halo depend on its eccentricity, see RDS [28]:

$$
\begin{gathered}
R=\left(\frac{15}{3 A_{3}(e)\left(1-e^{2}\right)^{2 / 3}}\right)^{1 / 2}\left(\frac{g}{4 \pi G m^{2}}\right)^{1 / 2}, \\
\lambda=\frac{6}{5 \sqrt{5}} \frac{\arcsin e}{e} t\left(1+\frac{e}{t} \frac{A_{3}(e)\left(1-e^{2}\right)^{1 / 2}}{\arcsin (e)}\right)^{1 / 2},
\end{gathered}
$$

with the $t$-parameter $t \equiv T /|W|$, a measure of rotational support, given by (see also LRS [20])

$$
t(e)=3 /\left(2 e^{2}\right)-1-3 \sqrt{1-e^{2}} /(2 e \arcsin (e)),
$$

and $A_{3}(e)=2 / e^{2}-2 \sqrt{1-e^{2}} \arcsin (e) / e^{3}$ for this model. On the other hand, a compressible, irrotational RiemannS ellipsoid of polytropic index $n=1$ must be prolate, i.e. its semi-axes fulfill $a_{1} \geq a_{3} \geq a_{2}$, and the eccentricities are given by $e_{1}=\left(1-\left(a_{2} / a_{1}\right)^{2}\right)^{1 / 2}$ and $e_{2}=\left(1-\left(a_{3} / a_{1}\right)^{2}\right)^{1 / 2}$. In that case, the expressions for the mean radius and spin parameter

$$
R=R_{0} g\left(e_{1}, e_{2}\right)^{-1 / 2}, \quad \lambda=\lambda\left(e_{1}, e_{2}\right)
$$
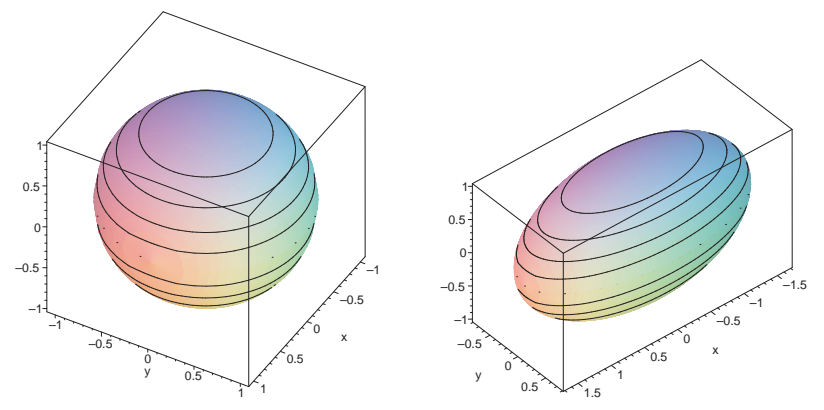

FIG. 1. Halos rotating about the $z$-axis for $a_{3}=1$ and $\lambda=0.05$ : Maclaurin spheroid having $e=0.302$ (left-handplot) and irrotational Riemann-S ellipsoid having $\left(e_{1}, e_{2}\right)=$ (0.881; 0.797) (right-hand-plot); see also RDS [28].

with $R_{0}$ in (25) and $g\left(e_{1}, e_{2}\right)$ and $\lambda\left(e_{1}, e_{2}\right)$ functions of the eccentricities, are very cumbersome and we refer to RDS [28], equ.(101) for more details.

For both models, the characteristic size - the mean radius - depends on the particle parameters exactly the same way as in the non-rotating case, namely $R \sim \sqrt{g} / \mathrm{m}$. The effect of the rotation is thus only to change the overall multiplicative factor of this dependence as seen from (27) and (30). By fixing $\lambda=(0.01,0.05,0.1)$, we can solve $\lambda=\lambda(e)$ in (28) and $\lambda=\lambda\left(e_{1}, e_{2}\right)$ in (30), respectively, for the eccentricities. Fig 1 shows two illustrative examples of our rotating halo models.

We were able in RDS [28] to derive the generalization to equ.(24) for the case of the $(n=1)$-polytropic Riemann-S ellipsoid analytically, assuming the ellipsoidal approximation of LRS [20]. Accordingly, the ellipsoidal density profile reads

$$
\rho^{E}(\tilde{q})=\rho_{c}^{E} \operatorname{sinc}\left[\tilde{q}\left(1-e_{1}^{2}\right)^{1 / 6}\left(1-e_{2}^{2}\right)^{1 / 6} g\left(e_{1}, e_{2}\right)^{1 / 2}\right]
$$

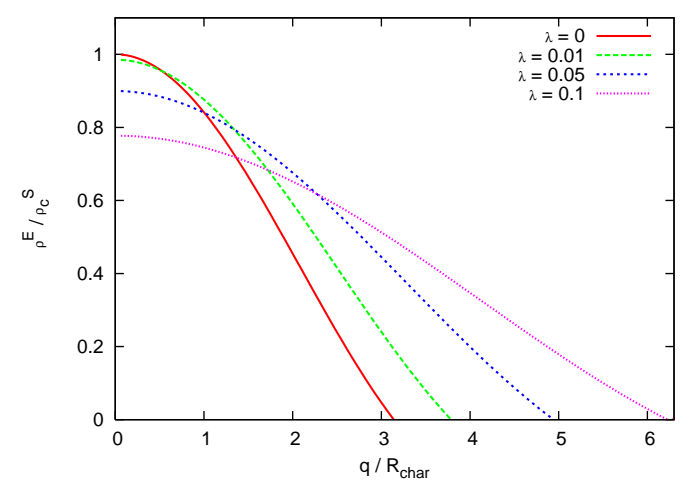

FIG. 2. Density profiles of the (vortex-free) $(n=$ 1)-polytropic Riemann-S ellipsoidal halos having $\lambda=$ $(0.01,0.05,0.1)$, according to equ. (31). The profile of the spherical halo, equ.(24), is added for comparison (solid curve). The densities are all normalized to $\rho_{c}^{S}=1 ; q=\tilde{q} R_{\text {char }} \equiv$ $\tilde{q}\left[g /\left(4 \pi G m^{2}\right)\right]^{1 / 2}$. The locus of the outer surface, where the density vanishes, increases with $\lambda$; see also RDS [28]. 
with

$$
\tilde{q}^{2}=\left[x^{2}+y^{2} /\left(1-e_{1}^{2}\right)+z^{2} /\left(1-e_{2}^{2}\right)\right]\left(\pi / R_{0}\right)^{2},
$$

$\rho_{c}^{E}$ the central density of the ellipsoid, and $R_{0}$ in (25). It is plotted for different $\lambda$ in Fig, 2 .

The virial theorem (22) not only provides the above relationships for the mean radius and spin-parameter of a halo, but also relates the DM particle parameters in a characteristic way: it can be shown that the corresponding formulae are

$$
\frac{m}{m_{H}}=\left(\frac{5}{8 A_{3}(e)\left(1-e^{2}\right)^{2 / 3}}\right)^{1 / 2} \sqrt{\frac{g}{g_{H}}}
$$

for Maclaurin spheroidal halos, and

$$
\frac{m}{m_{H}}=\frac{\pi}{\sqrt{8}} g\left(e_{1}, e_{2}\right)^{-1 / 2} \sqrt{\frac{g}{g_{H}}}
$$

for Riemann-S ellipsoidal halos, see RDS [28]. That is, virialized rotating halos, according to either model, will lie on a straight line in $(\log m, \log g)$-space, whose slope is completely determined by the eccentricities or mean radius of a given halo. These relationships can be found in Fig 4 and Fig 5 respectively. From those and the condition $g / g_{H} \gg 2$, it follows that, in the TYPE II regime, $m / m_{H} \gg 1$, as it must be for $\lambda_{d e B} \ll R_{0}$.

In [28], we also determined the conditions for the formation of a central quantum vortex in a given rotating halo. The minimum amount of angular momentum necessary to form a singly-quantized, axisymmetric vortex in the center of a halo with total number of particles $N=M / m$ is given by

$$
L_{Q M} \equiv N \hbar,
$$

which, for a given $\lambda$ and $L$ becomes a minimum condition on the particle mass. The relationship is linear for both halo models, i.e.

$$
m / m_{H}=f\left(e_{1}, e_{2}\right) L / L_{Q M},
$$

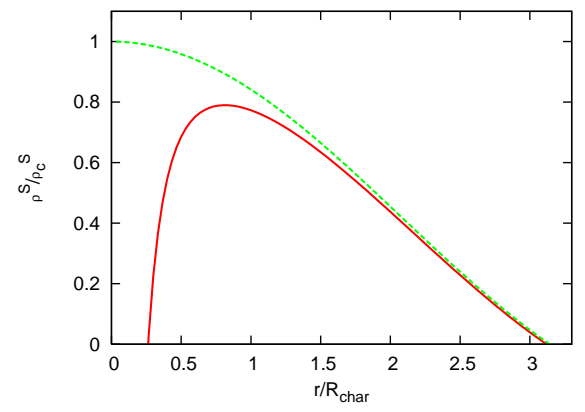

FIG. 3. Density profile of a spherical halo with vortex in the center (solid line) which transitioned from a Riemann-S ellipsoidal halo having $\lambda=0.05$. The profile of the spherical halo with $L=0$ and no vortex (dashed line) is added for comparison; $R_{\text {char }}$ as in Fig. 2. See also RDS [28]. with $f\left(e_{1}, e_{2}\right)$ denoting here another function depending only on the eccentricities (see equ.(108) and (109) in [28]). In order to find a criterion which is, not only necessary, but also sufficient, we determined the DM particle parameters for which the central vortex is energetically favored, i.e. the conditions which make the halo with vortex have less energy than a rotating, but otherwise vortex-free halo. For this purpose, we consider two limiting cases: Model $A$ describes a halo with a high enough angular momentum that $L \gg L_{Q M}$, i.e. the central vortex is essentially considered to be only a small perturbation of the total angular momentum $L$ of the halo. For this case, we use Maclaurin spheroids as our halo model. Model B, on the other hand, assumes a given halo has just enough angular momentum to form one quantum vortex, i.e. $L=L_{Q M}$. We use the irrotational, $(n=1)$-polytropic Riemann-S ellipsoids for this case. In this case, once the central vortex is energetically favored, its formation takes up all of the angular momentum, leaving a spherical halo with vortex, see Fig 3 for an example.

Despite differences in the assumed halo equilibrium models, the conclusions are the same for Model $A$ and Model $B$ : vortex formation requires a minimum particle mass $m \geq m_{\text {crit }}$ and a minimum particle self-interaction coupling strength $g \geq g_{\text {crit }}$. For any $(m, g)$ pair which satisfies $m \geq m_{\text {crit }}$ and $g \geq g_{\text {crit }}$ at a given $\lambda$, this pair will also favor vortex formation for any larger value of $\lambda$. Our main results are summarized in Fig,4 and 5 , which are independent of halo size, respectively, while Table [II and III list physical units for $m_{\text {crit }}, g_{\text {crit }}$ and the corresponding vortex size, for the cases of a typical dwarf galaxy (DG) and a dwarf spheroidal galaxy (dSph), according to Table【 Since we fix $\lambda$ and $L=L_{Q M}$ for the case of Model B, the respective particle parameters $\left(\left(\mathrm{m} / \mathrm{m}_{H}\right)_{\text {crit }},\left(\mathrm{g} / \mathrm{g}_{H}\right)_{\text {crit }}\right)$ are uniquely determined via equ.(35) and (33), respectively (see also Fig. 5 for those numbers). It turns out that for all $\lambda$ considered, vortex formation is favored for those parameters. We interpret this to mean that, for these same $\lambda$-values, if $L / L_{Q M}>1$, instead, (i.e. $m / m_{H}>\left(m / m_{H}\right)_{c r i t}$, according to (35)), vortex formation will also be favored, as long as $g / g_{H}>\left(g / g_{H}\right)_{c r i t}$. On the other hand, for halos with $L<L_{Q M}$, the particle parameters must satisfy $m / m_{H}<\left(m / m_{H}\right)_{c r i t}$ and $g / g_{H}<\left(g / g_{H}\right)_{c r i t}$, respectively, so they will not form vortices, but can still be modeled as Riemann-S ellipsoids. (This assumes, of course, that $g / g_{H} \gg 2$, so they are still in the TYPE II regime.)

We shall comment on other previous work on vortices in related models. Silverman \& Mallet [32] give heuristic arguments for vortex formation, but do not derive the critical conditions or their consequences for the DM particle parameters. Yu \& Morgan [40] show that vortex lattices can provide flat rotation curves for galaxies. Kain and Ling [17], on the other hand, find approximate solutions for the density profile of a nonrotating, sphericallysymmetric halo with a single vortex that contains all the 
TABLE II. Lower bounds on the boson mass and selfinteraction coupling strength for vortex formation in TYPE II BEC-CDM halos with a given spin parameter $\lambda$ in Model $A$. $\xi_{\text {max }}$-values denote the upper bounds for the corresponding vortex core radius.

\begin{tabular}{llll}
\multicolumn{4}{c}{ Dwarf Galaxy } \\
\hline \hline$\lambda$ & $m_{\text {crit }}[\mathrm{eV}]$ & $g_{\text {crit }}\left[\mathrm{eV} \mathrm{cm}{ }^{3}\right]$ & $\xi_{\text {max }}[\mathrm{kpc}]$ \\
\hline 0.01 & $1.04 \cdot 10^{-21}$ & $2.30 \cdot 10^{-58}$ & 0.03 \\
0.05 & $1.67 \cdot 10^{-22}$ & $5.74 \cdot 10^{-60}$ & 0.20 \\
0.10 & $7.33 \cdot 10^{-23}$ & $1.02 \cdot 10^{-60}$ & 0.47 \\
\hline \hline \multicolumn{4}{c}{ Dwarf Spheroidal Galaxy } \\
\hline \hline$\lambda$ & $m_{\text {crit }}[\mathrm{eV}]$ & $g_{\text {crit }}\left[\mathrm{eV} \mathrm{cm^{3 }}\right]$ & $\xi_{\text {max }}[\mathrm{kpc}]$ \\
\hline 0.01 & $3.30 \cdot 10^{-20}$ & $2.30 \cdot 10^{-57}$ & $3.13 \cdot 10^{-3}$ \\
0.05 & $5.28 \cdot 10^{-21}$ & $5.74 \cdot 10^{-59}$ & 0.02 \\
0.10 & $2.32 \cdot 10^{-21}$ & $1.02 \cdot 10^{-59}$ & 0.05 \\
\hline \hline
\end{tabular}

TABLE III. Lower bounds on the boson mass and selfinteraction coupling strength for vortex formation in TYPE II BEC-CDM halos with a given spin parameter $\lambda$ in Model B. $\xi_{\max }$-values denote the upper bounds for the corresponding vortex core radius.

\begin{tabular}{llll}
\multicolumn{4}{c}{ Dwarf Galaxy } \\
\hline \hline$\lambda$ & $m_{\text {crit }}[\mathrm{eV}]$ & $g_{\text {crit }}\left[\mathrm{eV} \mathrm{cm}{ }^{3}\right]$ & $\xi_{\text {max }}[\mathrm{kpc}]$ \\
\hline 0.01 & $1.50 \cdot 10^{-22}$ & $3.59 \cdot 10^{-60}$ & 0.25 \\
0.05 & $3.20 \cdot 10^{-23}$ & $1.53 \cdot 10^{-61}$ & 1.21 \\
0.10 & $1.69 \cdot 10^{-23}$ & $3.87 \cdot 10^{-62}$ & 2.41 \\
\hline \hline \multicolumn{4}{c}{ Dwarf Spheroidal Galaxy } \\
\hline \hline$\lambda$ & $m_{\text {crit }}[\mathrm{eV}]$ & $g_{\text {crit }}\left[\mathrm{eV} \mathrm{cm} \mathrm{cm}^{3}\right]$ & $\xi_{\text {max }}[\mathrm{kpc}]$ \\
\hline 0.01 & $4.75 \cdot 10^{-21}$ & $3.59 \cdot 10^{-59}$ & 0.02 \\
0.05 & $1.01 \cdot 10^{-21}$ & $1.53 \cdot 10^{-60}$ & 0.12 \\
0.10 & $5.34 \cdot 10^{-22}$ & $3.87 \cdot 10^{-61}$ & 0.24 \\
\hline \hline
\end{tabular}

angular momentum for the TYPE II case (as in Fig. 3, solid curve). Their estimates for the viable parameter space of particle mass for the Andromeda galaxy is in agreement with our more precise results for the Milky Way, see RDS [28].

Our results strongly suggest that vortices can only form in BEC-CDM with a sufficiently large, positive selfinteraction coupling strength. Vortex formation in axion DM without self-interaction, which has been claimed by Sikivie \& Yang [31], seems thus not viable according to our results. However, for TYPE II halos, i.e. those for which $m / m_{H} \gg 1$ and $g / g_{H} \gg 2$, vortex formation is favored for the range of halo spin parameters found in the CDM model, for a large portion of the BEC particle parameter space. For example, for $\lambda=0.05$, Model $B$ yields $\left(\mathrm{m} / \mathrm{m}_{H}\right)_{\text {crit }}=9.49$ and $\left(\mathrm{g} / \mathrm{g}_{H}\right)_{\text {crit }}=68.00$, while Model $A$ yields $\left(m / m_{H}\right)_{c r i t}=49.52$ and $\left(g / g_{H}\right)_{c r i t}=2549.24$ (see Fig. 4 and 5). Since the presence of a vortex causes the DM density profile to drop inside the vortex core, diminishing it altogether at the very center, the effect of vortices must be seriously considered whenever the TYPE II regime is studied as a model to describe galactic halo dynamics. At the critical values for vortex forma- tion and a little above, the effect of the vortex could be observable, as the $\xi_{\text {max }}$-values in Table $\amalg$ and $\amalg$ suggest. However, while it is true that the vortex becomes increasingly favored for large $g$ (at fixed $\lambda$ ), its size relative to the halo size decreases, i.e. for large enough $g$ the influence of the vortex will yet again diminish.

\section{Influence on baryonic substructures}

The foregoing sections exemplified some distinctive characteristics of Bose-Einstein-condensed DM models as compared to standard collisionless CDM. A further interesting topic is the detailed dynamics of baryonic substructure within galactic halos made of BEC-CDM. While this may likewise provide evidence for the presence or absence of this form of DM, it has hardly been considered in the previous literature.

Goodman [13] seems to be the first to discuss the consequences of TYPE II, as a superfluid, for a rotating galactic bar. This discussion has been continued in Appendix B of Slepian \& Goodman [34], with the result that the drag on the bar can be smaller than estimated for standard CDM, providing a potential remedy to a problem which plagues the latter.

Lora at el. [22], on the other hand, have recently studied the dynamic survival of cold gas clumps and globular clusters in the dSph galaxies of Ursa Minor and Fornax, respectively, in both TYPE I and TYPE II regimes. The survival of those structures within the lifetime of these galaxies of known mass provides valuable constraints on the allowed boson mass and self-interaction coupling strength. For TYPE I, their calculations favor a boson mass between $0.3 \cdot 10^{-22} \mathrm{eV}<\mathrm{m}<10^{-22} \mathrm{eV}$, while $m$ can be larger for TYPE II at fixed coupling strength, their constraint reading $\mathrm{g} / \mathrm{m}^{2} \gtrsim 8 \cdot 10^{-19} \mathrm{~cm}^{3} / \mathrm{eV}$ (expressed in our units), corresponding to core sizes larger

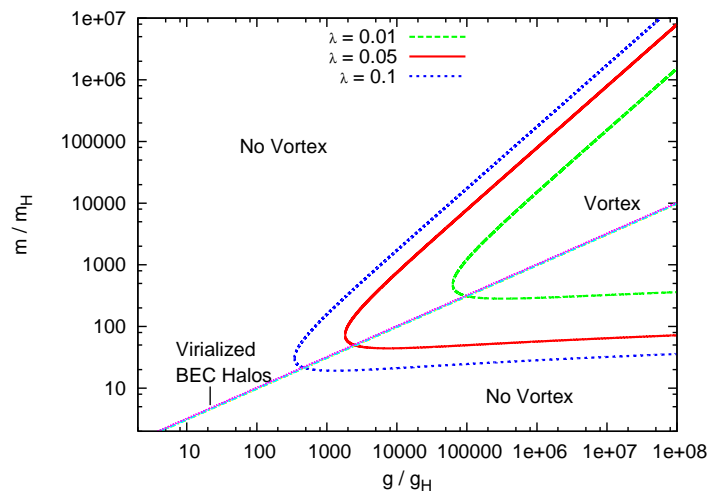

FIG. 4. Model A: Curves within which vortex formation is energetically favored in the dimensionless BEC-CDM particle parameter space $\left(\mathrm{m} / \mathrm{m}_{H}, g / g_{H}\right)$ for halos with spin parameter $\lambda=0.01,0.05,0.1$, respectively. Straight lines for the same $\lambda$ - values: halos fulfilling virial equilibrium, according to equ. [32); see also RDS [28]. 
than about $R \gtrsim 0.7 \mathrm{kpc}$. The first result can be understood given the limits in Table 【, while the second result is in accordance with previous studies, including our own ones as presented in Section [II. In both cases, Lora et al. 222] find viable parts of the parameter space of BECCDM which can explain the observations, while others can be definitely excluded.

The study of the dynamics of baryonic 'test bodies' like globular clusters or giant molecular clouds, which are large enough to feel the influence of the subtleties in the DM distribution, while still being much smaller than the DM halo, will provide useful constraints on BEC-CDM for our own Galaxy. There is valuable and interesting work to be done in future studies of this problem.

Finally, we note that galaxies are often observed to harbor supermassive black holes at their centers, with associated quasar luminosity which supports the idea that the black hole mass grew primarily by baryonic accretion. One might ask if BEC-CDM is consistent with this phenomenon, since its fluid behavior might imply a much higher accretion rate than that of collisionless CDM particles (c.f. Shapiro \& Teukolsky [30]). The gravitational collapse of non-self-interacting scalar field dark matter onto a central Schwarzschild black hole in a sphericallysymmetric space-time has been considered, for example, by Barranco et al. [6] and references therein, to determine if it is possible for DM halos in this model to survive for cosmological time scales. As we discussed in Section IIIB, however, halos have angular momentum. For accretion onto a central black hole to occur, this angular momentum must be overcome, which usually depends upon a dissipative process involving some form of viscosity to transfer that angular momentum outward. As a frictionless superfluid, BEC-CDM halos, however, cannot do this. Moreover, if a vortex is formed at the center, the lower density there will further inhibit accretion.

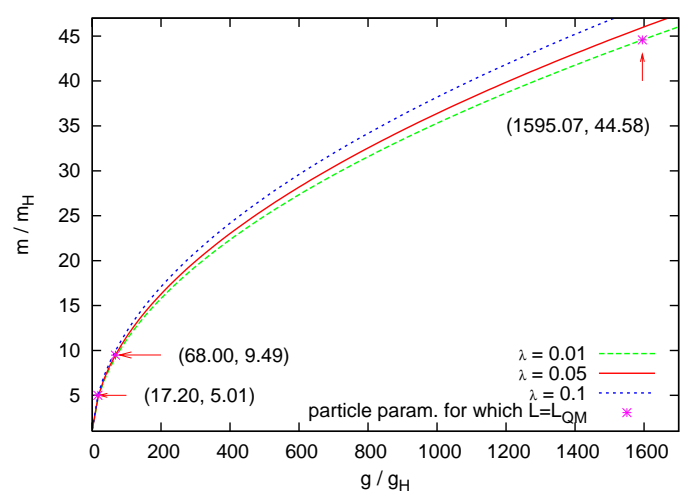

FIG. 5. Model B: Dimensionless BEC-CDM particle parameter space $\left(m / m_{H}, g / g_{H}\right)$ (no log-scale !) for halos with spin parameter $\lambda=0.01,0.05,0.1$, respectively, fulfilling virial equilibrium, according to equ.(33). For each $\lambda$, dots denote those critical particle parameters for which $L=L_{Q M}$; see also $\operatorname{RDS}[28]$.

\section{BOUNDS ON PARTICLE MASS AND COUPLING STRENGTH}

We have seen that the equilibrium size of isolated, hydrostatic BEC-CDM halo structures is predominantly governed either by quantum pressure, equ.(8), or by the polytropic pressure due to the collective, repulsive selfinteraction of the DM particles, equ.(9), for $g \lesssim g_{H}$ or $g \gtrsim g_{H}$, respectively. By opposing gravity, quantum and self-interaction pressures each prevent structure from forming on small scales, thereby imposing lower limits on the size of structures we can expect to find in a BECCDM universe. The lower limit set by quantum pressure is $\lambda_{d e B}$ in (10), evaluated using the halo virial velocity (which also characterizes the bulk mass motions at its formation time). Since the smallest halos also have the smallest virial velocities, the most stringent limit results if we require $\lambda_{d e B} \lesssim R$ for the smallest halos. This, in turn, imposes the lower limit on $m$, see Table 【. For a given $m$ that satisfies this lower limit, the coupling strength $g$ must not exceed the value such that the radius of the polytrope supported by self-interaction pressure, $R_{0}$ in (25) or the respective generalizations for rotating halos in (27) and (30), exceeds the size of the smallestscale structure.

The same requirement that the characteristic size $R_{0}$ not exceed the size $R$ of the smallest halos can also be used to place an upper limit on particle mass $m$, if we can establish an upper bound on the 2-body scattering cross section per unit particle mass, $\sigma / m$, from some other argument. Slepian and Goodman [34] have argued that upper bounds on $\sigma / m$ for the elastic-scattering particles in the SIDM model, based upon comparing that model to astronomical observation, should apply to BEC-CDM, as well. The interpretation of the Bullet cluster observations, for example, as a nearly collisionless merger of two cluster-sized halos has been found to limit $\sigma / m$ for SIDM halos to $(\sigma / m)_{\max }<1.25 \mathrm{~cm}^{2} / \mathrm{g}$, according to Randall et al. 27]. We can relate $\sigma / m$ for TYPE II BEC-CDM to the characteristic size $R_{0}$ in equ.(25) according to

$$
\frac{\sigma}{m}=\frac{8 G^{2}}{\pi^{3} \hbar^{4}} R_{0}^{4} m^{5}
$$

as pointed out by Slepian and Goodman [34]. In order for (36) to be applicable, the particle mass must be such that $m / m_{H} \gg 1$, in which case RDS [28] noted that (36) can be rewritten in fiducial units related to the size $R$ and mass $M$ of a given halo or halo core, as follows:

$$
\begin{gathered}
\frac{\sigma}{m}=2.094 \cdot 10^{-95}\left(\frac{m}{m_{H}}\right)^{5} \times \\
\times\left(\frac{R}{1 \mathrm{kpc}}\right)^{3 / 2}\left(\frac{M}{10^{8} M_{\odot}}\right)^{-5 / 2} \frac{\mathrm{cm}^{2}}{\mathrm{~g}}
\end{gathered}
$$

(where 'g' here means 'grams', not coupling strength). This shows that $\sigma / m$ is very much smaller than 
$(\sigma / m)_{\max } \sim 1 \mathrm{~cm}^{2} / \mathrm{g}$, unless the particle mass is many orders of magnitude larger than the lower bounds, $m / m_{H}=1$, in Table【. We can place an upper bound on $m$, in fact, if we replace $R_{0}$ in equ.(36) by $R$, the smallest halo (or halo core) size which must be produced, and replace $\sigma / m$ by $(\sigma / m)_{\max }$, to write

$$
m<\left(\frac{\pi^{3} \hbar^{4}}{8 G^{2}}\right)^{1 / 5}\left(\frac{\sigma}{m}\right)_{\max }^{1 / 5} R^{-4 / 5} .
$$

If we take as our fiducial units $R=1 \mathrm{kpc}$, and $(\sigma / m)_{\max }=1 \mathrm{~cm}^{2} / \mathrm{g}$, this gives

$$
m<9.193 \cdot 10^{-4} \mathrm{eV} / \mathrm{c}^{2} .
$$

To be self-consistent, we must check if our assumption is valid that BEC-CDM remains a pure condensate without thermalizing during virialization. If the relaxation time for particle collisions to establish thermodynamic equilibrium at the halo virial temperature is less than a Hubble time $\left(\sim 10^{17} \mathrm{sec}\right)$, our assumption would break down. In that case, Slepian and Goodman 34 determined that BEC-CDM halos in the TYPE II regime would have cores surrounded by isothermal envelopes of non-condensate, which would yield density profiles in disagreement with observed halos (unless $\sigma / m$ exceeds $(\sigma / m)_{\max } \sim 1 \mathrm{~cm}^{2} / \mathrm{g}$ by orders of magnitude). It can be shown, however, that if $(\sigma / m)_{\max } \sim 1 \mathrm{~cm}^{2} / \mathrm{g}$, then the relaxation time for achieving thermodynamic equilibrium is, indeed, more than a Hubble time, so thermodynamic equilibrium is not achieved for particle masses which obey inequality (39). Hence, this upper limit is a self-consistent one. Apparently, there is quite a large range of particle mass allowed between these upper and lower limits.

\section{BEYOND THE POLYTROPIC SIZE LIMIT}

Nevertheless, this requirement that $R_{0}<R$ for the smallest halos suggests there is a problem for this model in its simplest form, if observations require us to accommodate the formation of objects as small as the smallest dwarf spheroidal galaxies, while at the same time serving to explain the flattening of the density profiles in the cores of much larger galaxies. The remedy suggested by Slepian \& Goodman [34], where BEC-CDM cores in the TYPE II regime are enshrouded by isothermal envelopes of non-condensate, was unfortunately shown not to work by the same authors.

We envisage a different scenario to overcome the size limit given by the equilibrium model as follows: we assume that (25) characterizes the size of the inner core of larger halos, which grow larger as a result of continuous infall at the time of halo formation. Let us sketch this here in more detail. It can be shown that a classic, spherical top-hat model for the collapse and virialization of a cosmological density perturbation applied to BEC-CDM in the Einstein-de Sitter universe yields a post-collapse virialized object with radius $R_{T H}$ and uniform density $\rho_{0}$, given by

$$
\frac{R_{T H}}{r_{t a}}=\frac{2}{3} \text { and } \frac{\rho_{0}}{\rho_{t a}}=\left(\frac{3}{2}\right)^{3},
$$

where $r_{t a}$ and $\rho_{t a}$ are the radius and density of the tophat at the time of turn-around (i.e. maximum expansion). Then, the (virial) radius is determined from (22) as

$$
R_{T H}=\sqrt{\frac{15}{2}}\left(\frac{g}{4 \pi G m^{2}}\right)^{1 / 2}
$$

Not surprisingly, this $R_{T H}$ depends upon $g$ and $m$ in the same way the polytrope radius $R_{0}$ does, so the implication is that halos of different mass must form from cosmological fluctuations that collapse at different times, in order that $\rho_{t a}$ is different. Unfortunately, more massive halos require higher $\rho_{t a}$, since $R_{T H}$ is independent of halo mass, but higher $\rho_{t a}$ requires collapse at earlier times, which reverses the hierarchical structure formation history expected for CDM.

The crucial idea in overcoming this undesirable feature is the realization that equ.(41) neglects any internal kinetic energy of the virialized object. In fact, it can be shown that the virial radius grows beyond $R_{T H}$, once an (effective) kinetic term has been added, even though the regime of TYPE II is retained. Determining the form and the physical meaning of this additional kinetic part, and how it can advance the above description to provide a successful model for halo formation and structure will be the subject of a forthcoming paper. An immediate observation is the fact that the effective kinetic energy $K_{\text {eff }}$ should not simply be a constant factor times $W$ or $U_{S I}$, since this will only increase the virial radius by a fixed factor times $R_{T H}$, as can be easily shown. In fact, we have seen that the inclusion of uniform rotation has had exactly this effect, see equ.(27, 30). Instead, we will pursue the following idea: the BEC-CDM fluid must undergo oscillations during the process of virialization due to its inherently quantum-mechanical nature. In fact, the results of work by Khlopov et al.[18] and Gúzman \& Ureña-López [14] lend support to this idea.

Suppose $K_{\text {eff }}$ describes wave motions. For the general argument outlined here, it is sufficient to consider a non-vanishing bulk velocity $\mathbf{v}$ as before, i.e. $K_{\text {eff }}=T=$ $\int \frac{\rho_{0}}{2} \mathbf{v}^{2} d V>0$. Since $\rho_{0}=$ const., we assume that the gross average in the form of $T=\frac{3}{2} M \sigma_{v}^{2}$ with velocity dispersion $\sigma_{v}^{2}=\frac{1}{3}\left\langle\mathbf{v}^{2}\right\rangle$, will capture the overall kinetic contribution due to wave motions. Additionally, in order for the virial radius to depend on halo mass and time of collapse $t_{\text {coll }}$, as they do for standard CDM, we require the top-hat density to be a fixed fraction of the background density at $t_{\text {coll }}$, that is, $\rho_{0}=A \rho_{b, c o l l}$, with the constant $A$ depending on the background cosmology, e.g. $A \simeq 178$ for standard CDM in a flat, matter-dominated universe. The corresponding total mass is then $M=\frac{4}{3} \pi R^{3} A \rho_{b, \text { coll }}$, 
which yields a radius

$$
R\left(M, t_{\text {coll }}\right)=\left(\frac{3 M}{4 \pi A} \frac{1}{\rho_{b, \text { coll }}}\right)^{1 / 3} .
$$

Inserting this expression for $R$ into (22) with $K_{Q}=0$ and solving for the unknown velocity dispersion, we get

$\sigma_{v}^{2}\left(M, t_{\text {coll }}\right)=\frac{(36 \pi)^{1 / 3}}{15} G M^{2 / 3}\left(A \rho_{b, \text { coll }}\right)^{1 / 3}-\frac{g}{2 m^{2}} A \rho_{b, \text { coll }}$.

To interpret this result, we observe the following: For $g=0$, the last term vanishes and we recover the standard case, but for increasing $g>0$, this term makes $\sigma_{v}$ smaller. We can calculate the minimum mass for which $\sigma_{v}=0$, resulting in

$$
\begin{gathered}
M_{\text {min }}=\left(\frac{15}{(36 \pi)^{1 / 3} G}\right)^{3 / 2} A\left(\frac{g}{2 m^{2}}\right)^{3 / 2} \rho_{b, \text { coll }}= \\
=\frac{4}{3} \pi A \rho_{b, \text { coll }} R_{T H, 0}^{3}
\end{gathered}
$$

with $R_{T H, 0}$ given by (41). Thus, the smallest mass halo has the minimum size of $R_{T H, 0}$ by construction. The particle parameters may now be chosen such that $M_{\min }$ corresponds to the smallest observed galaxies, as well as to the cores of large galaxies, e.g. $M_{\min } \simeq 10^{8} M_{\odot}$. Larger halos then follow the relationship (42) and have a non-vanishing velocity dispersion due to internal wave motion, according to (43). This guarantees that BECCDM halos of mass $M>M_{\text {min }}$ would share the massradius relation of halos in the standard CDM model, if halos of a given mass $M$ typically collapse at the same time as they do for standard CDM.

\section{ACKNOWLEDGMENTS}

TRD would like to thank Tonatiuh Matos and Claudia Moreno and the organizing committee for their kind hospitality at the IV International Meeting on Gravitation and Cosmology, Guadalajara, Mexico, May 21-25, 2012. This work was supported in part by U.S. NSF grants AST-0708176, AST-1009799 and NASA grants NNX07AH09G, NNG04G177G, NNX11AE09G to PRS. TRD also acknowledges support by the Texas Cosmology Center of the University of Texas at Austin.
[1] K. Ahn, P.R. Shapiro, MNRAS 363, 1092 (2005)

[2] M. Alcubierre, F.S. Guzmán, T. Matos, D. Núñez, L.A. Ureña-López, P. Wiederhold, Class.Quant.Grav. 19, 5017 (2002)

[3] A. Arbey, J. Lesgourgues, P. Salati, Phys.Rev.D 68, 023511 (2003)

[4] A. Arvanitaki, S. Dimopoulos, S. Dubovsky, N. Kaloper, J. March-Russell, Phys.Rev.D 81, 123530 (2010)

[5] J. Barnes, G. Efstathiou, ApJ 319, 575 (1987)

[6] J. Barranco, A. Bernal, J.C. Degollado, A. Diez-Tejedor, M. Megevand, M. Alcubierre, D. Núñez, O. Sarbach, Phys.Rev.D 84, 083008 (2011)

[7] C.G. Böhmer, T. Harko, JCAP 06, 025 (2007)

[8] S.M. Carroll, Phys.Rev.Lett. 81, 3067 (1998)

[9] S. Chandrasekhar, Ellipsoidal Figures of Equilibrium, Yale Univ.Press, New Haven, CT (1969)

[10] P.H. Chavanis, Phys.Rev.D 84, 043531 (2011)

[11] P.H. Chavanis, L. Delfini, Phys.Rev.D 84, 043532 (2011)

[12] M. Colpi, S.L. Shapiro, I. Wasserman, Phys.Rev.Lett. 57, 2485 (1986)

[13] J. Goodman, New Astronomy 5, no.2, 103 (2000)

[14] F.S. Guzmán, L.A. Ureña-López, 2004, Phys.Rev.D 69, 124033 (2004)

[15] U. Günther, A. Zhuk, Phys.Rev.D 56, 6391 (1997)

[16] W. Hu, R. Barkana, A. Gruzinov, Phys.Rev.Lett. 85, $1158(2000)$

[17] B. Kain, Y. Ling, Phys.Rev.D 82, 064042 (2010)

[18] M.Y. Khlopov, B.A. Malomed, Y.B. Zeldovich, MNRAS 215, 575 (1985)

[19] J. Koda, P.R. Shapiro, MNRAS 415, 1125 (2011)

[20] D. Lai, F.A. Rasio, S.L. Shapiro, ApJ Suppl. 88, 205
(1993)

[21] J.-W. Lee, S. Lim, JCAP 7, 01 (2010)

[22] V. Lora, J. Magaña, A. Bernal, F.J. Sánchez-Salcedo, E.K. Grebel, JCAP 011, 02 (2012)

[23] E. Madelung, Z.für Phys. 40, 322 (1927)

[24] T. Matos, L.A. Ureña-López, Phys.Rev.D 63, 063506 (2001)

[25] M. Membrado, A.F. Pacheco, J. Sañudo, Phys.Rev.A 39, 4207 (1989)

[26] P.J.E. Peebles, ApJ 534, 2, L127 (2000)

[27] S.W. Randall, M. Markevitch, D. Clowe, A.H. Gonzalez, M. Bradač, ApJ 679, 1173 (2008)

[28] T. Rindler-Daller, P.R. Shapiro, MNRAS 422, 135 (2012)

[29] A. Schneider A., R.E. Smith, A.V. Macciò, B. Moore, MNRAS 424, 684 (2012)

[30] S.L. Shapiro, S.A. Teukolsky, Black Holes, White Dwarfs, and Neutron Stars, John Wiley \& Sons, Inc., New York, NY (1983)

[31] P. Sikivie, Q. Yang, Phys.Rev.Lett. 103, 111301 (2009)

[32] M.P. Silverman, R.L. Mallet, Gen.Rel.Grav. 34, 633 (2002)

[33] S.J. Sin, Phys.Rev.D 50, 3650 (1994)

[34] Z. Slepian, J. Goodman, MNRAS 427, 839 (2012)

[35] A. Suárez, T. Matos, MNRAS 416, 87 (2011)

[36] I.I. Tkachev, Phys.Lett.B 261, 289 (1991)

[37] L.A. Ureña-López, F.S. Guzmán, Phys.Rev.D 68, 024023 (2003)

[38] X.Z. Wang, Phys.Rev.D 64, 124009 (2001)

[39] T.-P. Woo, T. Chiueh, ApJ 697, 850 (2009)

[40] R.P. Yu, M.J. Morgan, Class.Quant.Grav. 19, L157 (2002) 